\title{
EFECTO PREDESINFECTANTE DEL OXICLORURO DE COBRE SOBRE HONGOS CONTAMINANTES EN SEGMENTOS NODALES DE Guadua angustifolia KUNTH, PARA SU ESTABLECIMIENTO IN VITRO
}

\author{
PRE-DISINFECTANT EFFECT OF COPPER OXYCHLORIDE ON FUNGAL CONTAMINANTS IN \\ NODAL SEGMENTS OF Guadua angustifolia KUNTH, FOR IN VITRO ESTABLISHMENT
}

Rodolfo López ${ }^{1}$

Laboratorio de Biotecnología. Corporación Autónoma Regional del Quindío. Colombia. rlopez@uniquindio.edu.co

\section{RESUMEN}

En el establecimiento in vitro de tejidos vegetales, se reporta la condición de aplicar protocolos de desinfección, con poca información acerca de procesos de predesinfección; en las diferentes etapas de micropropagación de Guadua angustifolia, se cuenta con reportes de contaminación por hongos y bacterias, estando los hongos más ligados a la etapa de establecimiento, mientras que la presencia de bacterias se registra en todas las fases in vitro. Para el establecimiento in vitro de explantes de guadua, en el Laboratorio de Biotecnología Vegetal, de la Corporación Autónoma Regional del Quindío, Colombia, se procedió a evaluar el fungicida comercial Oxicloruro de Cobre aplicado como pretratamiento de desinfección de segmentos nodales de guadua, seguido de un protocolo de desinfección ya establecido, que consistió en alcohol del $90 \%$ por 3 min, seguido de inmersión en $\mathrm{NaClO}$ del $3 \%$ por $10 \mathrm{~min}$; la predesinfección se realizó mediante lavado con detergente comercial, en agua destilada estéril por $20 \mathrm{~min}$; seguidamente se sometieron los diferentes segmentos nodales (juvenil, maduro y diferenciado) a inmersión durante 30 y 60 min en soluciones de los productos comerciales: oxicloruro de cobre y Agrodyne, y su mezcla. Los menores índices de contaminación por hongos, se lograron con segmentos nodales juvenil y maduro, sometidos a oxicloruro de cobre, mientras que el segmento nodal juvenil presentó mayor contaminación por bacterias.

Palabras clave: Tejidos vegetales, protocolos, predesinfección, segmento nodal, establecimiento. 


\begin{abstract}
In in vitro establishment of plant tissues, we report the implementation status of disinfection protocols, with little information about pre-disinfection processes after explants were selected, according to species. At different stages of micropropagation of Guadua angustifolia there are reports of fungi and bacteria contamination, being the one with fungus more related to the establishment phase, while the presence of bacteria was recorded at all in vitro stages. For this reason, at the Biotechnology Laboratory, of the Autonomous Regional Corporation of Quindío, Colombia, we proceeded to evaluate different pretreatment disinfection of nodal segments on bamboo, accompanied by a disinfection protocol already established, consisting of $90 \%$ alcohol for $3 \mathrm{~min}$, followed by immersion in $\mathrm{NaClO}$ of $3 \%$ for $10 \mathrm{~min}$. the pre-disinfection was carried out by commercial detergent wash in sterile distilled water for $20 \mathrm{~min}$. then the different nodal segments (youth, mature and differentiated) were exposed to solutions of commercial products: copper oxychloride and Agrodyne and it's mix for 30 min and 60 min. The lower rates of fungal contamination were obtained with young and mature nodal segments subjected to copper oxychloride, while the juvenile nodal segments showed higher bacterial contamination.
\end{abstract}

Keywords: plant tissue, protocols, disinfection, nodal segment, establishment.

\section{INTRODUCCIÓN}

Los bambúes son plantas con gran diversidad de formas, con estructuras morfológicas especializadas en la propagación, tales como rizoma, culmo, y yema, entre otras; el rizoma es un eje segmentado que se convierte en el soporte de la planta, con gran importancia en el sistema de absorción, con las siguientes estructuras: a) Cuello del rizoma; b) primordio de raíces; c) raíces adventicias; d) yema (1).

En diferentes regiones colombianas, el cultivo de la guadua ha permitido el desarrollo de importantes actividades ambientales, económicas y sociales, gracias a sus propiedades físico-mecánicas, que la hacen selecta entre los bambúes.

La propagación tradicional de G. angustifolia puede ocasionar daños mecánicos que degeneran en deterioro fitosanitario de la plantación, lo que lo que ha llevado a evaluar nuevas alternativas de multiplicación, entre las cuales destaca la micropropagación (2).

Por tanto, la micropropagación vegetal de G. angustifolia es una alternativa importante a tener en cuenta en diferentes programas de siembra; para ello, se han estado evaluando diferentes técnicas de multiplicación in vitro, como lo reportan algunos autores (3-7).

En las fases de la micropropagación vegetal, la contaminación por hongos, bacterias y levaduras es un problema grave, con dificultades para su manejo. Las pérdidas de material causadas por la contaminación, se convierten en limitante para el éxito de cultivo in vitro y representan pérdidas notables en tiempo y económicas, haciendo necesarias las prácticas de desinfección superficial de los explantes (8-11). Por tanto, es imprescindible el estudio de métodos que conduzcan a la eliminación de los contaminantes microbianos presentes en el cultivo de tejidos vegetales (12-14).

El producto comercial oxicloruro de cobre $(\mathrm{OxiCu})$ se ha utilizado en protocolos de predesinfección de explantes vegetales para su establecimiento in vitro; se cuenta con reportes de Nirmal et al. (15), con trabajos de micropropagación de segmentos nodales de la especie Murraya koenigii; también Nirmal et al. (16), utilizando segmentos nodales del árbol Cinnamomum camphora; ambos trabajos registraron la exposición de los segmentos nodales con $\mathrm{OxiCu} 0.3 \%$, durante $20 \mathrm{~min}$ y 45 min respectivamente; aunque no muestran los resultados alcanzados, sí enfatizan sobre la utilización de $\mathrm{OxiCu}$, como predesinfectante de los explantes.

En la predesinfección de rizomas de Heliconia sp. para su establecimiento in vitro, Marulanda e Isaza (17) aplicaron el producto comercial Agrodyne, en concentraciones de 0.5 a $1.0 \mathrm{ml}$ en $100 \mathrm{ml}$ de agua, por $60 \mathrm{~min}$, alcanzando desinfecciones superiores al $60 \%$.

Los bambúes son especies que presentan altos índices de contaminación en las fases de micropropagación; algunos trabajos recomiendan la aplicación de protocolos de predesinfección con fungicidas comerciales; en trabajos de establecimiento in vitro realizados con Bambusa vulgaris, Hernández (18) utilizó los fungici- 
das Kasumin y Kilol como elementos importantes en la predesinfección de los meristemos apicales y axilares, con resultados efectivos.

Thakur y Sood (19), aplicaron el fungicida Bavistin a explantes multinodales del Bambú Dendrocalamus hamiltonii, mediante ciertas técnicas de predesinfección, alcanzando descontaminaciones superiores, comparadas con los materiales que no se sometieron a predesinfección.

El cultivo in vitro de segmentos nodales G. angustifolia, a partir de chusquines, presenta un alto grado de contaminación por hongos y bacterias, afectando las diferentes fases en su micropropagación, causando enormes pérdidas en la investigación, aun teniendo en cuenta la desinfección que puede proporcionar el $\mathrm{NaClO}$ a los explantes $(4-7 ; 13,14,20)$.

Jiménez et al. (4), probaron el efecto predesinfectante del fungicida Benomyl, a razón de $2.0 \mathrm{mg}$ por $100 \mathrm{ml}$ de agua durante $10 \mathrm{~min}$, para el establecimiento in vitro de segmentos nodales de $G$. angustifolia, con una contaminación superior al 58\%, donde los explantes sin tratamiento con este fungicida, mostraron una contaminación del 88\%.

López (5) evaluó la acción del producto comercial Agrodyne aplicado sobre plantas madre (chusquín) de G. angustifolia, como preventivo de la contaminación in vitro de sus explantes; los resultados para la disminución contaminante por bacterias fue positivo, aunque no así para el control de hongos. Sin embargo, las bacterias hicieron su aparición en posteriores fases de la micropropagación, como multiplicación y/o enraizamiento de vitroplantas de guadua.

En trabajos de micropropagación de G. angustifolia, Marulanda et al. (5) reportaron el establecimiento del $31.9 \%$ de segmentos nodales del tercio basal, con una brotación inferior al 3\%. Ramírez et al. (14), registraron contaminación por hongos, superior al 50\% para segmentos nodales de los tercios medio y basal; aunque para López (5) los segmentos nodales del tercio apical presentaron bajos índices de contaminación, no recomienda su utilización para el establecimiento in vitro, debido a que exhibieron los menores porcentajes de brotación.

El objetivo del presente trabajo consistió en disminuir la contaminación por hongos, en el establecimiento in vi- tro de segmentos nodales de guadua (juvenil, maduro y diferenciado), mediante la utilización de los fungicidas oxicloruro de cobre $(\mathrm{OxiCu})$ y Agrodyne, y su mezcla, durante $30 \mathrm{~min}$ y $60 \mathrm{~min}$, para definir un protocolo de predesinfección, previo a la desinfección con $\mathrm{NaClO}$, para programas de multiplicación in vitro de G. angustifolia, que permita su establecimiento in vitro con bajos niveles de contaminación por hongos.

\section{MATERIALES Y MÉTODOS}

\section{Selección de explantes}

La investigación se llevó a cabo en el Laboratorio de Biotecnología, del Centro Nacional para el Estudio del Bambú-Guadua, de la Corporación Autónoma Regional del Quindío (CRQ). Los chusquines de guadua de 3 meses de edad permanecieron bajo condiciones de invernadero, como plantas madre; las ramas multinodales, con 4 a 6 segmentos nodales, se transportaron al laboratorio en bolsas plásticas esterilizadas.

Cada segmento nodal fue cortado con aproximadamente $1.0 \mathrm{~cm}$ de longitud, y se categorizó según la madurez fisiológica de su yema: A. Yema indiferenciada; B. Primordio de yema; C. Yema diferenciada (Figura 1.).

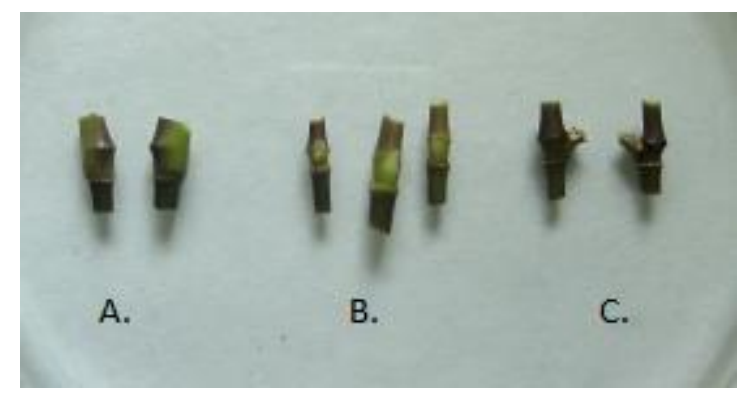

Figura 1. Segmentos nodales de G. angustifolia: A. Yema indiferenciada; B. Primordio de yema; C. Yema diferenciada.

\section{Predesinfección}

Los explantes se lavaron en solución de agua destilada estéril más detergente comercial, por 20 min; seguidamente se expusieron a los tratamientos de predesinfección de los fungicidas comerciales: OxiCu (100 mg en $100 \mathrm{ml}$ de agua), Agrodyne ( $1 \mathrm{~mL}$ en $100 \mathrm{~mL}$ de agua) y la mezcla de ellos en las mismas proporciones; los tratamientos fueron: T1. OxiCu por $30 \mathrm{~min}$; T2. OxiCu por $60 \mathrm{~min}$; T3. Agrodyne por $30 \mathrm{~min}$; T4. Agrodyne por 60 min; T5. Mezcla de OxiCu y Agrodyne por 60 min; T6. Tratamiento control, sin exposición de los explantes a predesinfección (Tabla 1). 
Tabla 1. Tratamientos de predesinfección de segmentos nodales de G. angustifolia según su yema (indiferenciada, primordio, diferenciada), con los productos OxiCu y Agrodyne por 30 y $60 \mathrm{~min}$.

\begin{tabular}{lll}
\hline TRATAMIENTO & EXPLANTE & PRODUCTO/Tiempo \\
\hline T 1A & Indiferenciada & OxiCu/30 min \\
T 1B & Primordio & OxiCu/30 min \\
T 1C & Diferenciada & OxiCu/30 min \\
T 2A & Indiferenciada & OxiCu/60 min \\
T 2B & Primordio & OxiCu/60 min \\
T 2C & Diferenciada & OxiCu/60 min \\
T 3A & Indiferenciada & Agrodyne/30 min \\
T 3B & Primordio & Agrodyne/30 min \\
T 3C & Diferenciada & Agrodyne/30 min \\
T 4A & Indiferenciada & Agrodyne/60 min \\
T 4B & Primordio & Agrodyne/60 min \\
T 4C & Diferenciada & Agrodyne/60 min \\
T 5A & Indiferenciada & OxiCu+Agrodyne/60 min \\
T 5B & Primordio & OxiCu+Agrodyne/60 min \\
T 5C & Diferenciada & OxiCu+Agrodyne/60 min \\
T 6A & Indiferenciada & Testigo \\
T 6B & Primordio & Testigo \\
T 6C & Diferenciada & Testigo \\
\hline
\end{tabular}

Seguidamente en cabina de flujo laminar, en todos los tratamientos, los segmentos nodales fueron tratados con alcohol del $90 \%$ por $3 \mathrm{~min}$, y luego se expusieron a solución de $\mathrm{NaClO}$ industrial del 3\%, por 10 min; luego se realizaron tres enjuagues con agua destilada estéril.

Para el desarrollo del presente trabajo, se utilizó el medio de cultivo MS basal propuesto por Murashige y Skoog (21), suplementado con $3.0 \mathrm{mg} \mathrm{l}^{-1}$ de la citoquinina BAP, y $30 \mathrm{gr} \mathrm{l}^{-1}$ de glucosa; se ajustó el $\mathrm{pH}$ a 6.0 , mediante la adición de gotas de $\mathrm{KOH} 1 \mathrm{~N}$; luego se agregaron $2.8 \mathrm{~g} / \mathrm{L}$ del gelificante comercial Culturegel, en agitación constante; los envases tipo gérber se sirvieron con $20 \mathrm{ml}$ del medio, para luego llevarse a condiciones de autoclave con $121{ }^{\circ} \mathrm{C} \mathrm{y} 15 \mathrm{psi}$, por $20 \mathrm{~min}$. En cada envase se inoculó el segmento nodal de manera individual, para lo que se emplearon 10 recipientes por tratamiento, con 10 repeticiones.

\section{Evaluación de la predesinfección:}

Los explantes se mantuvieron por seis semanas en sala de crecimiento, con 12 horas de fotoperíodo, y $24 \pm 2$ ${ }^{\circ} \mathrm{C}$. El efecto predesinfectante del OxiCu se evaluó según el número de segmentos nodales que no presentaron contaminación por hongos, durante las seis semanas de cultivo (Figura 2).

Para ello, se diseñó un experimento de bloques completamente aleatorizados, donde cada bloque correspondió a cada una de los explantes inoculados, bajo un arreglo factorial de dos factores controlados: segmento nodal (juvenil, maduro y diferenciado) de Guadua angustifolia y método de desinfección (5 tratamientos de pre- desinfectante). La variable dependiente fue la fracción (con transformación $\operatorname{Arc} \sin \sqrt{x}$ ) de explantes que sobrevivieron, y de explantes contaminados por hongos, después de seis semanas de cultivo.

El análisis estadístico se realizó mediante el paquete "Statistica for WINDOWS", versión 6.0 (StatSoft, 2001).

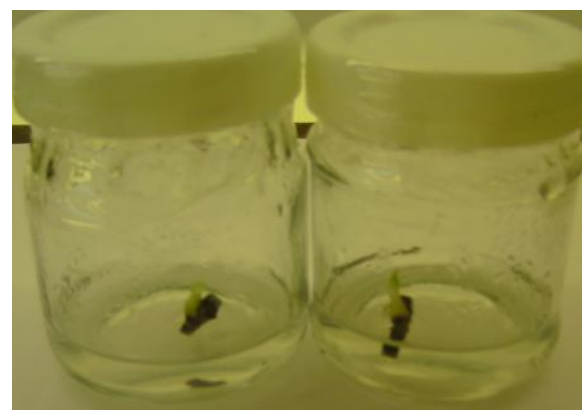

Figura 2. Brotación de segmentos nodales de guadua libres de contaminación.

\section{RESULTADOS}

\section{Desinfección de segmentos nodales:}

La Tabla 2 presenta los resultados de contaminación por hongos de los diferentes segmentos nodales (juvenil, maduro y diferenciado) de G. angustifolia en condiciones in vitro, durante seis semanas; se registró interacción entre tratamiento de predesinfección y tipo de explante ( $\mathrm{P}=0.0005)$; la prueba de LSD de comparación de medias, señaló que los segmentos nodales con yema indiferenciada y primordio de yema con los tratamientos T1A, T1B, T2A, T2B, T3A, T3B, T4A Y T4B, registraron menor porcentaje de contaminación por hongos $\mathrm{y}$ 
difieren significativamente de los demás tratamientos.

Tabla 2. Contaminación por hongos de diferentes segmentos nodales de G. angustifolia, según su yema (indiferenciada, primordio, diferenciada) con los productos OxiCu y Agrodyne por 30 y $60 \mathrm{~min}$.

\begin{tabular}{lll}
\hline TRATAMIENTO & EXPLANTE & HONGOS \\
\hline T 1A & Indiferenciada & $0.4585 \mathrm{EF}$ \\
T 1B & Primordio & $0.4868 \mathrm{EF}$ \\
T 1C & Diferenciada & $0.8870 \mathrm{~A}$ \\
T 2A & Indiferenciada & $0.4069 \mathrm{EF}$ \\
T 2B & Primordio & $0.4301 \mathrm{EF}$ \\
T 2C & Diferenciada & $0.7863 \mathrm{ABC}$ \\
T 3A & Indiferenciada & $0.4069 \mathrm{EF}$ \\
T 3B & Primordio & $0.4301 \mathrm{EF}$ \\
T 3C & Diferenciada & $0.7854 \mathrm{ABC}$ \\
T 4A & Indiferenciada & $0.3785 \mathrm{~F}$ \\
T 4B & Primordio & $0.4636 \mathrm{EF}$ \\
T 4C & Diferenciada & $0.7451 \mathrm{BCD}$ \\
T 5A & Indiferenciada & $0.5100 \mathrm{E}$ \\
T 5B & Primordio & $0.6606 \mathrm{D}$ \\
T 5C & Diferenciada & $0.7241 \mathrm{BCD}$ \\
T 6A & Indiferenciada & $0.6819 \mathrm{CD}$ \\
T 6B & Primordio & $0.7081 \mathrm{BCD}$ \\
T 6C & Diferenciada & $0.8870 \mathrm{~A}$ \\
\hline
\end{tabular}

Letras iguales no difieren estadísticamente, según Tukey $(\mathrm{p}<0.05)$

Los mayores índices de contaminación (superiores al $72 \%$ ) están asociados a los segmentos nodales con yema diferenciada, mientras que los segmentos nodales con yema indiferenciada y primordio de yema presentaron índices inferiores al 49\% de contaminación, cuando fueron sometidos a predesinfección con los productos comerciales OxiCu y Agrodyne, sin mostrar resultados exitosos cuando se mezclaron los dos productos.

\section{DISCUSIÓN}

Los resultados del presente trabajo de investigación concuerdan con los reportes de varios autores, en la medida que el fungicida Oxicloruro de cobre $(\mathrm{OxiCu})$ se ha utilizado para disminuir la presencia de hongos en el cultivo de tejidos vegetales (15-16).

También se ha reportado con éxito la utilización del producto comercial Agrodyne para el control de la contaminación in vitro, de segmentos nodales de G. angustifolia (5) y para el control de contaminación de explantes de Heliconia sp. en condiciones in vitro (17).

Con respecto a la utilización de diferentes explantes de G. angustifolia, para su establecimiento en condiciones in vitro, López (5) y Marulanda et al. (7) reportaron menores índices de contaminación cuando utilizaron segmentos nodales apical (yema indiferenciad) y medio (primordio de yema), a la vez que la mayor contaminación la registraron con segmentos nodales basales (yema diferenciada).

\section{CONCLUSIONES}

En el establecimiento in vitro de Guadua angustifolia, para disminuir la contaminación causada por hongos, se recomienda la siembra de segmentos nodales que poseen primordio de yema, exponiéndolos a una predesinfección con oxicloruro de cobre del $0.1 \%$ por $60 \mathrm{~min}$; también se propone la predesinfección con el producto comercial Agrodyne utilizando $1.0 \mathrm{~mL}$ en $100 \mathrm{~mL}$ de agua, durante $60 \mathrm{~min}$, sin realizar la mezcla de ambos productos.

\section{AGRADECIMIENTOS}

El autor agradece a la Corporación Autónoma Regional del Quindío (CRQ), el apoyo brindado para la realización de la presente investigación. 


\section{BIBLIOGRAFÍA}

1. Giraldo E. Sabogal A. Una alternativa sostenible: La Guadua. Técnicas de cultivo y manejo. Corporación Autónoma Regional del Quindío. Optigraf. Armenia. Colombia. 2007. 192 pp.

2. Cruz H. Bambú Guadua. Guadua angustifolia Kunth. Bosques Naturales de Colombia. Plantaciones Comerciales en México. Editorial Gráficas Olímpica S.A. Pereira. Colombia. 2009. 691pp.

3. Marulanda M., Gutierrez L.G., Márquez M. Micropropagación de Guadua angustifolia Kunth. Actual Biol. 2005. 27 (82): 5-15.

4. Jiménez V., Castillo J., Tavares E., Guevara E. y Montiel M. In vitro propagation of the neotropical giant bamboo, Guadua angustifolia Kunth, through axillary shoot proliferation. Plant Cell, Tiss Orga. 2006; 86:389-395.

5. López R. Micropropagación de Guadua angustifolia Kunth en medio sólido y por Inmersión Temporal, y estudio de su desarrollo en campo. Trabajo de Grado Magíster. Universidad del Quindío, Universidad Tecnológica, Universidad de Caldas. Armenia, Colombia. 2007. 99 pp.

6. Acosta M., Alvarado Y., Cruz M., Roque B., Sánchez C., Leiva M., Freire M., García y., Pérez Z., Salabarría T., Tejeda M., González M., Hurtado O. Micobiota de plantas donadoras y contaminantes fúngicos del establecimiento in vitro de especies de bambúes. Biotecnología Vegetal. 2008; 8(1): 57-61.

7. Marulanda M.L., López A.M. Uribe M. Gutiérrez L.G. Biodiversidad y Biotecnología de la Guadua angustifolia Kunth. Editorial Publiprint Ltda. Dosquebradas, Colombia. 2010. 106 pp.

8. Digonzelli, P.; Díaz, L.; Bellone, S. C.; Latife, J.; Sosa, S. Diferentes dosis de PPM para controlar la contaminación bacteriana y sus efectos sobre el crecimiento in vitro de caña de azúcar en la etapa de mutiplicación. In: Enconto latino-Americano de Biotecnología Vegetal, 2001, Goiânia, GO. Resumos. Goiânia: REDBIO, 2001. p. 90.

9. Alvarado Y., Portal N., García L., Ramírez D., Martínez Y. Incidencia de Contaminantes Microbianos en la Micropropagación de la Caña de Azúcar. Biotecnología Vegetal. 2003; 3 (1): 31-36.

10. Digonzelli P., Diaz L., Carrizo S. Uso de PPM (Plant Preservative Mixture) para controlar contaminantes bacterianos en la multiplicación in vitro de la Caña de Azúcar. Rev. Fac. Agron.. 2005. 22:22-32.

11. Das M., Pal A. In vitro regeneration of Bambusa balcoa Roxb. Factors affecting changes of morphogenetic competence in axillary buds. Planta Cell, Tissue and Organ Culture. 2005. 81(1): 109-112.

12. Leifert C., Ritchie J. y Waites W. Contaminants of plant tissue and cell cultures. J. Microbiol Biotechn. Estados Unidos. 1991. 452-469.

13. Cruz M., García Y., Sánchez C., Alvarado Y, Acosta M., Roque B., Leiva M. y Freire M. Identificación y Control de Bacillus sp. Contaminante del Establecimiento in vitro de Guadua angustifolia Kunth. Biotecnología Vegetal. 2007. Vol 7, № 1:9-13.

14. Ramírez L.A., Castaño S.M., López R. Identificación de Bacterias que afectan el establecimiento in vitro de segmentos nodales de Guadua angustifolia Kunth. Rev. Invest. Univ. Quindío. 2009. № 19: 151-158.

15. Nirmal K., Anu A., Remashree A.B. y Praveen K. Micropropagation of curry leaf tree. Plant Cell, Tissue and Organ Culture. 2000. 61:199-203.

16. Nirmal K., Sajina A., Minoo D., John C.Z., Mini P.M., Tushar K.V., Rema J. y Ravindran P.N. Micropropagation of camphor tree (Cinnamomum camphora). Plant Cell, Tissue and Organ Culture. 2003. 74: 179-183.

17. Marulanda M.L. e Isaza L. Establecimiento in vitro de Heliconas con fines de producción masiva. Scientia et Technica UTP. 2004. Año X, N 26: 193-197.

18. Hernández A. Establecimiento in vitro de Bambusa vulgaris (Bambú amarillo). Trabajo de Grado de Ingeniería en Biotecnología. Centro de Investigación en Biotecnología. Instituto Tecnológico de Costa Rica. Costa Rica. 2001. 24 pp. http://bibliodigital.itcr.ac.cr:8080/xmlui/bitstream/handle/2238/34/ BJFIB200330.pdf? sequence $=1$ 
19. Thakur R. y Sood A. An efficient method for explant sterilization for reduced contamination. Plant Cell, Tiss Orga. 2006; 84:369-371.

20. Borges M., Ros C., Castellanos Y., Milanes S. y Velásquez R. Efecto de Diferentes Métodos de Desinfección en el Establecimiento in vitro de Guadua angustifolia Kunth. Biotecnología Vegetal. 2004. Vol. 4(4): 237-242.

21. Murashige T. y Skoog, F. A revised medium for rapid growth and bioassays with tobacco tissue cultures. Physiol. Plant. 1962. 15:473-497 